\title{
Cisplatin, Tegafur-Uracil and Leucovorin plus Mitomycin C: An Acceptably Effective and Toxic Regimen for Patients with Recurrent or Metastatic Nasopharyngeal Carcinoma
}

\author{
Chia-Hsun Hsieh ${ }^{1,2}$, Cheng-Lung Hsu ${ }^{1}$, Cheng-Hsu Wang ${ }^{3}$, Chaung-Chi Liaw ${ }^{1}$, Jen-Shi Chen ${ }^{1}$, \\ Hsien-Kun Chang ${ }^{1}$, Tsai-Shen Yang ${ }^{1}$, John Wen-Chen Chang ${ }^{1}$, Yung-Chang Lin ${ }^{1}$, Chi-Ting Liau ${ }^{1}$, \\ Ngan-Ming Tsang ${ }^{4}$, Joseph Tung-Chieh Chang ${ }^{4}$, Shu-Hang Ng${ }^{5}$, Hung-Ming Wang ${ }^{1}$
}

Background: This prospective phase II clinical trial evaluated the efficacy and toxicity of cisplatin, oral tegafur-uracil, leucovorin, and mitomycin $\mathrm{C}$ in patients with recurrent or metastatic nasopharyngeal carcinoma.

Methods: Patients with histologically proven non-keratinizing or undifferentiated nasopharyngeal carcinoma were prospectively enrolled from April 2002 to June 2005. Cisplatin $50 \mathrm{mg} / \mathrm{m}^{2}$ on day 1,22 and mitomycin $\mathrm{C} 6 \mathrm{mg} / \mathrm{m}^{2}$ on day 1 were administered. Oral tegafur-uracil $300 \mathrm{mg} / \mathrm{m}^{2} /$ day and oral leucovorin $60 \mathrm{mg}$ /day were given on day 1-14 and day 22-35, respectively. Each cycle was repeated every 6 weeks. Primary and secondary endpoints are response rate and toxic profiles with survivals, respectively.

Results: $\quad$ Twenty-two patients with the median age of 47 (35-69) years were enrolled in the study. Sixteen (72.7\%) patients had undifferentiated nasopharyngeal carcinoma. The regimen was well-tolerated by all patients with the exception of one patient (4.6\%) who experienced grade IV anorexia, and two patients $(9.1 \%)$ who had grade IV vomiting. There was no treatment-related death. The overall response rate was $59.1 \%$, including $3(13.6 \%)$ complete remissions.

The median duration of response was 15.9 months, the median time to tumor progression was 10.0 months, and the median overall survival was 16.0 months.

Conclusion: This outpatient chemotherapy regimen is acceptably effective and toxic among patients with recurrent or metastatic nasopharyngeal carcinoma.

(Biomed J 2013;36:229-236)

\section{At a Glance Commentary \\ Scientific background of the subject}

New chemotherapeutic agents and combinations are expected to improve the side effects and the response of conventional chemotherapy in recurrent/metastatic nasopharyngeal carcinoma (NPC). Safety, efficacy and convenience of use are all concerned for new design.

\section{What this study adds to the field}

This prospective phase II trial evaluated the efficacy and toxicity of cisplatin, oral tegafur-uracil, leucovorin, and mitomycin C in NPC. Results showed that this regimen is acceptably effective and safe to be performed in outpatient clinics. This work provides an important information of new combination (PULM) to the treatment for mNPC patients.

\section{Key words: cisplatin, head and neck neoplasms, leucovorin, mitomycin C, nasopharyngeal cancer, tegafur-uracil}

From the ${ }^{1}$ Division of Hematology-Oncology, Department of Internal Medicine, Chang Gung Memorial Hospital at Linkou, Chang Gung University College of Medicine, Taoyuan, Taiwan; ${ }^{2}$ Department of Chemical Engineering and Institute of Chemical and Materials Engineering, Chang Gung University, Taoyuan, Taiwan; ${ }^{3}$ Department of Diagnostic Radiology, Chang Gung Memorial Hospital at Linkou, Chang Gung University College of Medicine, Taoyuan, Taiwan; ${ }^{4}$ Department of Radiation Oncology, Chang Gung Memorial Hospital at Linkou, Chang Gung University College of Medicine, Taoyuan, Taiwan; ${ }^{5}$ Division of Hematology-Oncology, Department of Internal Medicine, Chang Gung Memorial Hospital at Keelung, Chang Gung University College of Medicine, Taoyuan, Taiwan Received: Jun. 6, 2012; Accepted: Aug. 30, 2012

Correspondence to: Dr. Hung-Ming Wang, 5, Fusing St., Gueishan, Taoyuan 333, Taiwan (R.O.C.). Tel: 886-3-3281200 ext. 8825; Fax: 886-3-3286697; E-mail: whm526@adm.cgmh.org.tw

DOI: $10.4103 / 2319-4170.113375$ 
$\mathrm{N}^{\mathrm{a}}$ asopharyngeal carcinoma (NPC) is uncommon in the United States and Western Europe; however, is very prevalent in Southeast Asian countries, including Taiwan. The overall incidence of NPC is only around 0.7 cases per 100,000 population per year in the United States; however, is as high as 15-30 cases per 100,000 population per year in Hong Kong. ${ }^{[1]}$ According to the cancer registry of Taiwan, NPC accounted for $2.02 \%$ of the cancer incidence with 1,558 incident cases in 2008 . The crude incidence rates were 9.99 per 100,000 per year for men, and 3.47 per 100,000 per year for women. ${ }^{[2]}$

Before 2008, many phase II clinical studies of chemotherapy for patients with recurrent or metastatic NPC demonstrated median response rates of approximately $45 \%$ to $80 \%$, with median survival ranging from 7.2 to 15 months. ${ }^{[3-7]}$ These active chemotherapeutic agents comprised of cisplatin, 5-fluorouracil (5-FU), doxorubicin, epirubicin, bleomycin, mitoxantrone, mitomycin $\mathrm{C}$, taxanes, gemcitabine, cetuximab and sorafenib. ${ }^{[8-16]}$ Among all of the combinations, cisplatin plus 5-FU is still the most commonly used regimen for NPC, because no phase III studies have found any other regimen to be superior. However, evidence showed that there is still a curative role for optimized chemotherapy combinations in patients with complete remission and in long-term survivors. ${ }^{[17]}$

Tegafur-uracil (UFT, UFUR, TTY Biopharm Co. Ltd, Taipei, Taiwan) is an oral preparation combining tegafur (5-fluorouracil prodrug) and uracil in a 1:4 ratio. This chemotherapeutic agent has a low toxicity profile, is well tolerated by most patients, can be easily combined with other chemotherapeutic agents, and is convenient to administer in outpatient settings. ${ }^{[18]}$ In gastrointestinal, lung, and breast cancers, Tegafur-uracil is often used as an alternative to 5-FU. ${ }^{[19-22]}$ In addition, we previously reported that a Tegafur-uracil-based chemotherapy regimen (cisplatin, Tegafur-uracil, leucovorin; PUL) was effective and less toxic for patients with head and neck squamous carcinoma (HNSCC). ${ }^{[23]}$ Mitomycin C (M) is active in hypoxic circumstances and helps against tumor cells in the G0 phase. In recent studies, tumor hypoxia is found to be common in NPC, and is associated with disease progression and resistance to therapy. ${ }^{[24]}$ Adding mitomycin $\mathrm{C}$ to a cisplatin-based chemotherapy regimen may help to overcome resistance conferred by hypoxia and eradicate tumor cells, even those that are not in the cell cycle. ${ }^{[16]}$ Since NPC appears to be highly sensitive to chemotherapy as well as radiotherapy, ${ }^{[25]}$ the non-overlapping toxicity profile of mitomycin C, cisplatin, and oral fluoropyrimidines warrants such a combination for NPC, especially for outpatient settings.

Therefore, we conducted this phase II trial to evaluate the efficacy and toxicity of cisplatin, Tegafur-uracil, leucovorin, and mitomycin C (PULM) as an outpatient chemotherapy regimen for patients with recurrent or metastatic NPC.

\section{METHODS}

Protocol of this prospective phase II clinical trial was approved by the institutional review board of the Chang Gung Memorial Hospital at Linkou and informed consent was obtained from all patients. From April 2002 to June 2005 , we enrolled patients aged $\geqq 18$ and $\leqq 70$ years with histologically proven nonkeratinizing poorly-differentiated or undifferentiated nasopharyngeal carcinoma, as defined by the World Health Organization (WHO). Under the Fleming one stage design, the sample size was estimated to be 42 patients with alpha (0.05), power $(0.90)$ and expected response rate of $69.5 \% .{ }^{[26]}$ All patients had Eastern Cooperative Oncology Group (ECOG) performance status scores ranging from 0 to 2 , and recurrent or metastatic diseases that had not been treated by radiotherapy or surgery with curative intent. Previously concurrent chemo-radiotherapy regimens for local disease were allowed with the exception of oral Tegafur-uracil or mitomycin $\mathrm{C}$ exposure. None of the patients enrolled in this study had received chemotherapy or radiotherapy within 4 weeks of entry. All enrolled patients had at least one measurable recurrent or distant metastatic lesion. A measurable lesion was defined as one measuring $20 \mathrm{~mm}$ by conventional techniques or $\geqq 10 \mathrm{~mm}$ by spiral computerized tomography scans in the longest dimension. Bone lesions are included for response evaluation in this trial because bone is the most common site of metastases of NPC. Prostate cancer project criteria were utilized in this trial for response evaluation of bone lesions. ${ }^{[27]}$ Adequate bone marrow and organ function was defined as: (a) an absolute neutrophil count $\geqq 1,500 / \mu \mathrm{L}$; (b) a platelet count $\geqq 100,000 / \mu \mathrm{L}$; (c) a serum creatinine $\leqq 1.5$ times the upper limit of normal (ULN) or 24-hour creatinine clearance $\geqq 60 \mathrm{ml} / \mathrm{min}$; (d) total bilirubin $\leqq 1.5 \times \mathrm{ULN}$ or $\leqq 5.0 \times$ ULN in patients with hepatic metastasis; and (e) serum aspartate aminotransferase and alanine transaminase $\leqq 2.5 \times$ ULN or $\leqq 5.0 \times$ ULN in patients with hepatic metastasis.

Exclusion criteria consisted of decompensated liver cirrhosis; clinically detectable peripheral neuropathy in Oxaliplatin Specific Neurological Scale grade $\geqq 2$; active cardiac disease within 6 months (e.g., angina, myocardial disease); active infection; history of another malignancy with the exception of successfully treated cervical or skin carcinoma; pregnancy or breast-feeding status; ongoing anticancer treatment; documented hypersensitivity to any component of the study drugs; and intestinal obstruction or malabsorption that may preclude absorption of Tegafur-uracil.

Eligible patients were treated with cisplatin, tegafur-uracil (UFUR), leucovorin, and mitomycin C (PULM regimen). Cisplatin $50 \mathrm{mg} / \mathrm{m}^{2}$ was administered intrave- 
nously in $500 \mathrm{ml}$ of $0.9 \%$ normal saline or $5 \%$ dextrose in $0.9 \%$ normal saline over 3 hours on day 1 and day 22 . Oral Tegafur-uracil $300 \mathrm{mg} / \mathrm{m}^{2} /$ day with oral leucovorin $60 \mathrm{mg} /$ day was given on day 1-14 and day 22-35. Mitomycin C $6 \mathrm{mg} / \mathrm{m}^{2}$ was administered intravenously on day 1 every 6 weeks, and did not exceed a total dose of $60 \mathrm{mg}$. Each course of therapy was defined as a 6-week treatment [Figure 1].

In patients with a neutrophil count $<1500 / \mu \mathrm{L}$ or platelet count $<1 \times 10^{5} / \mu \mathrm{L}$ on the day of scheduled chemotherapy, the treatment was postponed for a maximum of 4 weeks. Chemotherapy was discontinued if the neutrophil or platelet count did not recover. Tegafur-uracil was reduced by $50 \mathrm{mg} / \mathrm{m}^{2}$ if any grade IV hematologic toxicity or grade III non-hematologic toxicity occurred. The protocol was completely stopped if a third dose reduction of Tegafur-uracil was needed.

Tumor response was evaluated every 2 courses (12 weeks) according to the Definition of Objective Response in Solid Tumors (WHO criteria). When partial response (PR) or complete remission (CR) was achieved, the results were confirmed by imaging study one month later. In patients with stable disease, the status was confirmed by an imaging study at a minimum interval of 2 courses from the entry of study. Ultrasound or computed tomography scans or magnetic resonance imaging scans and nasopharyngoscopy were performed whenever any sign or symptom suggestive of disease progression was noted. Toxicity was graded according to the National Cancer Institute Common Toxicity Criteria (NCI-CTC) version 2.0. Treatment was discontinued if there was evidence of disease progression or unacceptable toxicity, or because of patient refusal or death from any cause.

\section{Endpoints and survival analysis}

The primary endpoint was response rate. Secondary endpoints were toxicity profile, time to tumor progression (TTP), duration of response, and overall survival (OS).

\begin{tabular}{|c|c|c|c|c|c|}
\hline$\frac{\text { Mitomycin C }}{6 \mathrm{mg} / \mathrm{m}^{2} \text { i.v. bolus }}$ & & & & & \\
\hline Cisplatin & & & Cisplatin & & \\
\hline $\begin{array}{l}50 \mathrm{mg} / \mathrm{m}^{2} \mathrm{i} . v . f \\
\text { for } 3 \text { hours }\end{array}$ & & & $\begin{array}{l}50 \mathrm{mg} / \mathrm{m}^{2} \mathrm{i} . v . f \\
\text { for } 3 \text { hours }\end{array}$ & & \\
\hline Tegafur/uracil 3 & $\mathrm{mg} / \mathrm{m}^{2} / \mathrm{d}$ & & Tegafur/urac & $00 \mathrm{mg} / \mathrm{m}^{2} / \mathrm{d}$ & \\
\hline $\begin{array}{l}\text { \& Leucovorin } 6 \\
\text { divided into } 4 \text { ti }\end{array}$ & $\begin{array}{l}\mathrm{ng} / \mathrm{d} \\
\text { les/d }\end{array}$ & Rest & $\begin{array}{l}\text { \& Leucovori } \\
\text { divided into }\end{array}$ & $\begin{array}{l}\mathrm{Omg} / \mathrm{d} \\
\mathrm{mes} / \mathrm{d}\end{array}$ & Rest \\
\hline D1 & D2 14 & D15 21 & D22 & D23 35 & D36 42 \\
\hline
\end{tabular}

Figure 1: Treatment schedule of study regimen.
TTP was defined as survival without disease progression or death from any cause. It was calculated from the first day of PULM administration to the date of progression or most recent follow-up date. OS was calculated from the first day of PULM treatment to the date of death or most recent follow-up date. Likewise, duration of response was calculated from the date of best response proven by imaging studies to the date of documented disease progression. Follow-up time was defined as duration from enrollment to the date of the most recent follow-up or death. All data of survivors were updated in February, 2012. Descriptive statistics were used to report clinical characteristics. All events were counted according to the intention-to-treat principle. The duration of response, TTP, and OS were estimated using the Kaplan-Meier method.

\section{RESULTS}

Forty-six patients were originally designed to be enrolled in this trial; however, only 22 patients were enrolled during the study period due to slow recruitment. Table 1 summarized the clinical and pathologic characteristics. The median age was 47 years (range: $35-69$ years) and

Table 1: Clinical and pathologic characteristics of the 22 patients

\begin{tabular}{|c|c|c|}
\hline & $n$ & $\%(N=22)$ \\
\hline \multicolumn{3}{|l|}{ Age } \\
\hline Median (range), years & $47(35-69)$ & \\
\hline \multicolumn{3}{|l|}{ Gender } \\
\hline Male & 16 & $72.7 \%$ \\
\hline Female & 6 & $27.3 \%$ \\
\hline \multicolumn{3}{|l|}{ Pathologic subtypes } \\
\hline Nonkeratinizing carcinoma & 6 & $27.8 \%$ \\
\hline Undifferentiated carcinoma & 16 & $72.7 \%$ \\
\hline \multicolumn{3}{|l|}{ Disease status at enrollment } \\
\hline Post-treatment locoregional recurrence & 1 & $4.5 \%$ \\
\hline Post-treatment distant recurrence & 10 & $45.5 \%$ \\
\hline Post-treatment local disease+distant recurrence & 3 & $13.6 \%$ \\
\hline Initial local disease+distant metastasis & 8 & $36.4 \%$ \\
\hline \multicolumn{3}{|l|}{ Performance status (ECOG) } \\
\hline 0 & 2 & $9.1 \%$ \\
\hline 1 & 17 & $77.3 \%$ \\
\hline 2 & 3 & $13.6 \%$ \\
\hline \multicolumn{3}{|l|}{ Sites of distant metastasis } \\
\hline Bone & 14 & $63.6 \%$ \\
\hline Liver & 13 & $59.1 \%$ \\
\hline Lung* & 7 & $31.9 \%$ \\
\hline Lymph node & 6 & $27.3 \%$ \\
\hline Brain+skull base invasion & 3 & $13.6 \%$ \\
\hline Spleen & 1 & $4.6 \%$ \\
\hline Soft tissue & 1 & $4.6 \%$ \\
\hline
\end{tabular}

Biomed J Vol. 36 No. 5 September - October 2013 
the male-to-female ratio was 2.67 (16:6). Sixteen (72.7\%) patients had undifferentiated carcinoma. The most common disease status was post-treatment distant recurrence $(n=10$, $45.5 \%)$. The sites of distant metastasis were mainly at bone $(n=14,63.6 \%)$ and liver $(n=13,59.1 \%)$.

The regimen was generally well tolerated. The most common toxicities were grade III leucopenia in 6 (27.3\%) patients, grade II neutropenia in $11(50.0 \%)$ patients, and grade I thrombocytopenia in $16(72.7 \%)$ patients. One $(4.6 \%)$ patient experienced grade IV anorexia and two $(9.1 \%)$ patients had grade IV vomiting. There was no treatment-related death in this trial [Table 2]. Dose modification was required in $3(13.6 \%)$ patients and one $(4.6 \%)$ of them quitted the trial because of prolonged thrombocytopenia for more than 4 weeks.

All enrolled patients received the PULM regimen for a median duration of 5.1 months (range: 1.1-11.3 months). The median number of cycles was 2.5 (range: 1-7). The rate of compliance was high (20 of 22 patients; $91 \%$ ). The two patients who did not comply with the scheduled treatments were due to personal reasons rather than toxicity. After a median follow-up of 17.3 months (range: 2.563.3 months), the best response achieved was complete response in $3(13.6 \%)$ patients, partial response in $10(45.5 \%)$ patients, and stable disease in $1(4.6 \%)$ patient [Table 3]. Among the responders, the median duration of response was 15.9 months (95\% confidence interval [CI]: 3.9-27.8). The median cycles of received chemotherapy between responders $(n=13)$ and non-responders $(n=9)$ were 5 and 2 , respectively ( $p=0.003$ ). Responders were observed to be associated with a long duration of response (15.9 months) and a median survival time of 19.1 months. No significant difference in survival time was found between responders and non-responders. $(p=0.16)$. Subgroup analysis revealed

Table 2: Drug-related toxicity profile

\begin{tabular}{lcccc}
\hline Toxicities $(N=22)$ & \multicolumn{4}{c}{ Grade (NCI-CTC version 2)* } \\
\cline { 2 - 5 } & Grade 1 & Grade 2 & Grade 3 & Grade 4 \\
\cline { 2 - 5 } & $n(\%)$ & $n(\%)$ & $n(\%)$ & $n(\%)$ \\
\hline Hematologic & $2(9.1 \%)$ & $9(40.9 \%)$ & $6(27.3 \%)$ & $0(0.0 \%)$ \\
Leucopenia & $5(22.7 \%)$ & $11(50.0 \%)$ & $2(9.1 \%)$ & $0(0.0 \%)$ \\
Neutropenia & $6(27.3 \%)$ & $9(40.9 \%)$ & $2(9.1 \%)$ & $0(0.0 \%)$ \\
Anemia & $16(72.7 \%)$ & $2(9.1 \%)$ & $1(4.6 \%)$ & $0(0.0 \%)$ \\
Thrombocytopenia & & & & \\
Gastrointestinal & $3(13.6 \%)$ & $1(4.6 \%)$ & $0(0.0 \%)$ & $0(4.6 \%)$ \\
Anorexia & $2(9.1 \%)$ & $3(13.6 \%)$ & $2(9.1 \%)$ & $0(0.0 \%)$ \\
Mucositis & $7(31.8 \%)$ & $2(9.1 \%)$ & $2(9.1 \%)$ & $0(0.0 \%)$ \\
Nausea & $3(13.6 \%)$ & $3(13.6 \%)$ & $0(0.0 \%)$ & $0(9.1 \%)$ \\
Vomiting & $4(18.2 \%)$ & $1(4.6 \%)$ & $2(9.1 \%)$ & $0(0.0 \%)$ \\
Diarrhea &
\end{tabular}

Abbreviation: NCI-CTC: National cancer institute-common toxicity criteria; *The number in this table was calculated by severest events experienced by patients that patients with initial metastasis tended to have better median TTP, OS, and duration of response than patients with recurrent distant metastasis. However, no statistical significance in those outcome variables was found $(p=0.224,0.986$ and 0.378 , respectively; data not shown). The Kaplan-Meier survival curves for TTP and OS were shown in Figure 2. Median TTP was 10.0 months (95\% CI: 6.9-13.0) and median OS was 16.0 months (95\% CI: 5.2-26.9). Long-term survival (> 60 months) was observed in 2 patients $(9.1 \%)$ with disease-free status.

\section{DISCUSSION}

In this phase II trial, the overall response rate was $59.1 \%$, the disease control rate was $63.6 \%$, the median duration of response was 15.9 months, the median time to tumor progression was 10.0 months, and the overall survival rate was 16.0 months. Comparing to previous trials historically [Table 4], ${ }^{[28-40]}$ acceptable duration of response, response rates and OS were observed in this trial. Results of previous trials focusing on different chemotherapy regimens for treatment of recurrent or metastatic NPC showed variable ranges of treatment response. Conventional cisplatin-based regimens had response rates ranging from $45 \%$ to $80 \%$; carboplatin-based regimens were shown to have response rates ranging from $30 \%$ to $75 \%$; taxanes alone achieved rates ranging from $21.7 \%$ to $36.7 \%$; and taxane-based combination regimens had response rates ranging from $27.5 \%$ to $86 \%$. Gemcitabine plus platinum seemed to have a better response rate $(63.6 \%$ to $86.0 \%$ ) and overall survival (15.0-22.0 months) than other regimens; however, there is no head-to-head comparison data among these regimens to date. Targeted therapies are novel

Table 3: Evaluation of response to the PULM regimen

\begin{tabular}{lcc}
\hline & $n$ & $\%(N=22)$ \\
\hline Follow-up time & & \\
$\quad$ Median (range), months & 17.3 & $(2.5-63.3)$ \\
Best response achieved $(n=22)$ & 3 & $13.6 \%$ \\
CR & 10 & $45.5 \%$ \\
PR & 1 & $4.6 \%$ \\
SD & 7 & $31.8 \%$ \\
PD & 1 & $4.6 \%$ \\
NE & & \\
Duration of response $(n=11) *$ & 15.9 & $(3.9-27.8)$ \\
Median (95\% CI), months &
\end{tabular}

Abbreviations: PULM: Cisplatin (P), tegafur-uracil (UFUR, U), leucovorin (L) and mitomycin C (M); CR: Complete remission; PR: Partial response; SD: Stable disease; PD: Progressive disease; NE: Not evaluated; CI: Confidence interval; *Two initial responders were censored (original responder number $=13$ ) in this analysis because they withdrew from trial as personal reasons without any evidence of progression. One patient requested transferring to neighboring local hospital for further treatment after first partial response was achieved. The other was withdrawn due to treatment-related prolonged thrombocytopenia without disease progression 


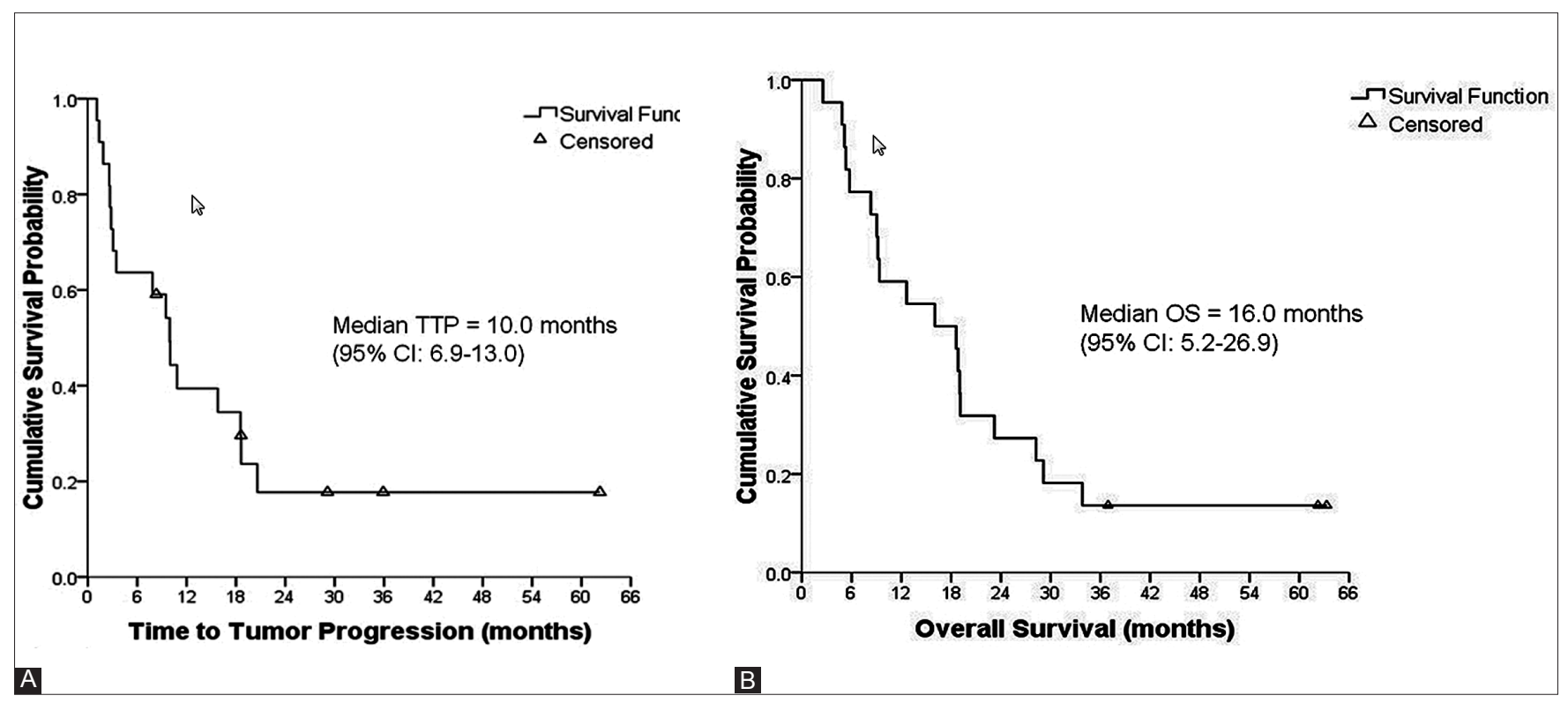

Figure 2: Demonstration of time to tumor progression and overall survival curve. Panel A shows median TTP was 10.0 months. Panel B indicates median OS was 16.0 months. (Abbreviation: CI: Confidence interval).

anti-cancer strategies; however, the response rates in those trials were quite low (40.7\% for sorafenib alone and $11.7 \%$ for cetuximab plus carboplatin). Most disappointingly, no complete remission was achieved in those trials. ${ }^{[8,14]} \mathrm{A}$ complete remission rate of $13.6 \%$ was noted in our trial whereas the average complete remission rate was approximately $7.0 \%$ in previous trials.

To the best of our knowledge, the duration of response in this study (15.9 months) was satisfactory compared historically to those in previous phase II trials (approximately 8 months). The plausible reason or mechanism is the anti-angiogenic effects of metronomic oral 5-FU, e.g., Tegafur-uracil or capecitabine, which have been widely discussed in many studies. In our study, results also showed statistically differences on total received cycles of chemotherapy between responders and non-responders (median cycles of 5 versus 2, respectively, $p=0.003$ by Student $t$ test). Responders to PULM regimen are found to be associated with a long duration of response (15.9 months) and survival time (19.1 months). However, the differences (total chemotherapy cycles, survivals and duration of response) between responders and non-responders in this trial are still probably due to selection bias from responses; for instance, patients who got responses would keep going on further chemotherapy and other non-responders would stop treatment. On the other hands, there are emerging studies suggesting that long-term continuous administration of tegafur-uracil for cancer control can inhibit the development of feeding blood vessels to tumors, thereby suppressing tumor growth and prolonging the duration of response. ${ }^{[41-43]}$ Similar results with prolonged TTP and OS have been obtained with oral capecitabine alone. ${ }^{[4]}$ Cisplatin plus capecitabine has also been shown to be a safe, convenient, and well-tolerated regimen, although hand-foot syndrome is an annoying and common side effect. ${ }^{[45]}$ Therefore, Tegafur-uracil-containing regimen might show the potential to maintain continuous tumor control with less skin toxicity.

Hematologic toxicity was a common but well-tolerated side effect in this trial. Three (13.64\%) patients experienced grade IV toxicity with anorexia/vomiting; however, toxicity resolved after dose modification. Only one patient dropped out of the trial because of prolonged ( $>4$ weeks) grade I thrombocytopenia. But the patient had liver cirrhosis history and synchronous splenic metastasis, making it difficult to determine whether the thrombocytopenia was due to the regimen or was secondary to underlying cirrhosis and splenic involvement. In addition, some investigators claim that platinum-based triplets have significant toxicities and the possibility of increasing toxicities becomes an alarming problem when use of platinum-based triplets or regimens containing more than 3 drugs. ${ }^{[1,46,47]}$ Hasbini et al., had ever reported the FMEP regimen (mitomycin C, 5-FU, epirubicin, and cisplatin) was reported to be associated with a high rate of toxicity as well as a mortality rate of $9 \% .{ }^{[48]}$ However, the dosages of mitomycin, cisplatin, and 5-FU in our trial were much lower than those used in studies that reported high rates of toxicity. The low dosages may explain why the toxicity in our trial was acceptable. Early dose modification or aggressive supportive management is needed to prevent severe morbidity.

However, few early withdrawals from this study were still found which needs to be proved not related to the toxicity of study drugs. After analysis, the main reasons 
Table 4: Summary of recent studies of chemotherapy for recurrent/metastatic NPC (platinum- and taxane-based)

\begin{tabular}{|c|c|c|c|c|c|c|c|c|}
\hline Author & Country & $N$ & Regimen & $\begin{array}{l}\text { ORR } \\
(\%)\end{array}$ & $\begin{array}{l}\mathrm{CR} \\
(\%)\end{array}$ & $\begin{array}{l}\mathrm{mDR} \\
(\mathrm{mo})\end{array}$ & $\begin{array}{l}\mathrm{mTTP} \\
(\mathrm{mo})\end{array}$ & $\begin{array}{l}\mathrm{mOS} \\
(\mathrm{mo})\end{array}$ \\
\hline \multicolumn{9}{|l|}{ Cisplatin-based } \\
\hline Boussen et al., 1991 ${ }^{[6]}$ & Algeria & 49 & Cisplatin $+5 \mathrm{FU}+$ Bleomycin & $86.0 \%$ & $20.0 \%$ & 4.0 & N/A & Mean: 25 \\
\hline Au et al., $1994^{[3]}$ & Singapore & 24 & Cisplatin $+5 \mathrm{FU}$ & $66.0 \%$ & $14.0 \%$ & N/A & 8.0 & 11.0 \\
\hline Chi et al., 1994[7] & Taiwan & 35 & Cisplatin $+5 \mathrm{FU}+\mathrm{LV}$ & $80.0 \%$ & $13.3 \%$ & N/A & N/A & 14.0 \\
\hline Azli et al., $1995^{[4]}$ & Fracne & 44 & Cisplatin+Bleomycin+Epirubicin & $45.0 \%$ & $20.0 \%$ & N/A & N/A & N/A \\
\hline Taamma et al., 1999[15] & France & 49 & $\begin{array}{l}\text { Cisplatin+5FU+Bleomycin+Epirub } \\
\text { icin (BEPF) }\end{array}$ & $78.0 \%$ & $39.1 \%$ & N/A & 9.0 & 15.0 \\
\hline Ngan et al., 2002[28] & Hong Kong & 44 & Cisplatin+Gemcitabine & $78.0 \%$ & $20.5 \%$ & Mean: 5.3 & 10.6 & 15.0 \\
\hline Chua et al., 2005 & Hong Kong & 19 & Cisplatin+Docetaxel & $62.5 \%$ & $6.3 \%$ & N/A & 5.6 & 12.4 \\
\hline Li et al., $2008^{[5]}$ & China & 48 & Cisplatin+Capecitabine & $62.5 \%$ & $6.3 \%$ & N/A & 7.7 & 13.5 \\
\hline Current work, 2012 & Taiwan & 22 & Cisplatin+UFUR+LV+Mitomycin C & $59.1 \%$ & $13.6 \%$ & 15.9 & 10.0 & 16.0 \\
\hline \multicolumn{9}{|l|}{$\begin{array}{l}\text { Taxane- and new } \\
\text { platinum-based regimen }\end{array}$} \\
\hline Yeo et al., $1996^{[30]}$ & Hong Kong & 42 & Carboplatin $+5 \mathrm{FU}$ & $38.0 \%$ & $17.0 \%$ & N/A & N/A & 12.1 \\
\hline Yeo et al., $1998^{[31]}$ & Hong Kong & 27 & Paclitaxel+Carboplatin & $59.0 \%$ & $11.0 \%$ & N/A & 6.0 & 13.9 \\
\hline Au et al., $1998^{[9]}$ & Singapore & 24 & Paclitaxel alone & $21.7 \%$ & $0.0 \%$ & 7.5 & 2.5 & 12.0 \\
\hline Tan et al., 1999 & Singapore & 32 & Paclitaxel+Carboplatin & $75.0 \%$ & $3.0 \%$ & N/A & 7.0 & 12.0 \\
\hline Ciuleanu et al., 2004 ${ }^{[33]}$ & Romania & 40 & Paclitaxel+Carboplatin & $27.5 \%$ & $7.5 \%$ & N/A & 3.5 & 11.5 \\
\hline Chan et al., 2005 $5^{[8]}$ & Hong Kong & 60 & Cetuximab+Carboplatin & $11.7 \%$ & $0.0 \%$ & N/A & 2.7 & 7.8 \\
\hline Leong et al., 2008 $8^{[34]}$ & Singapore & 28 & $\begin{array}{l}\text { Paclitaxel+Gemcitabine+Carboplat } \\
\text { in, then maintenance 5FU/LV }\end{array}$ & $86.0 \%$ & $11.0 \%$ & 8.0 & 8.1 & 22.0 \\
\hline Ngeow et al. $2010^{[11]}$ & Singapore & 40 & Docetaxel alone & $36.7 \%$ & $0.0 \%$ & N/A & 4.5 & 12.8 \\
\hline \multicolumn{9}{|l|}{ Gemcitabine-based regimen } \\
\hline Ma et al., 2002 $[10]$ & Canada & 32 & $\begin{array}{l}\text { Gemcitabine alone vs. } \\
\text { Gemcitabine+Cisplatin }\end{array}$ & $\begin{array}{l}36 \text { vs. } \\
64 \%\end{array}$ & $\begin{array}{l}6 \text { vs. } \\
14 \%\end{array}$ & 4.25 vs. 6 & $\begin{array}{c}5 \mathrm{vs} . \\
9.7\end{array}$ & $\begin{array}{l}\text { 1yr-OS: } 48 \% \\
\quad \text { vs. } 64 \%\end{array}$ \\
\hline Foo et al., 2002 $2^{[5]}$ & Singapore & 52 & $\begin{array}{l}\text { Gemcitabine alone (chemonaive vs. } \\
\text { pre-treated) }\end{array}$ & $28 / 48.1 \%$ & & $4 \% / 3.7 \%$ & $3.6 / 5.1$ & $7.2 / 10.5$ \\
\hline Wang et al., 2006 & Taiwan & 39 & Gemcitabine+Vinorelbine & $36.0 \%$ & $3.0 \%$ & 5.1 & 5.6 & 11.9 \\
\hline Zhang et al., 2008 ${ }^{[13]}$ & China & 32 & Gemcitabine alone & $43.8 \%$ & $0.0 \%$ & N/A & 5.1 & 16.0 \\
\hline Ma et al., 2009 & Hong Kong & 41 & Gemcitabine+Oxaliplatin & $63.6 \%$ & $2.4 \%$ & 6.8 & 8.9 & 19.6 \\
\hline \multicolumn{9}{|l|}{ Single agent or other regimen } \\
\hline Dugan et al., $1993^{[12]}$ & Hong Kong & 108 & Mitoxantrone alone & $25.0 \%$ & $5.0 \%$ & 4.7 & 2.7 & 13.1 \\
\hline Fandi et al., 1997[41] & Tunisia & 21 & 5FU (low-dose) continuous infusion & $25.0 \%$ & $10.0 \%$ & 15.0 & 4.0 & 10.0 \\
\hline Au et al., $1998^{[9]}$ & Singapore & 24 & Paclitaxol alone & $21.7 \%$ & $0.0 \%$ & 7.5 & 2.5 & 12.0 \\
\hline Poon et al., 2005 & Singapore & 28 & Irinotecan alone & $14.0 \%$ & $0.0 \%$ & N/A & 3.9 & 11.4 \\
\hline Chua et al., 2008 ${ }^{[44]}$ & Hong Kong & 59 & Capecitabine alone & $37.0 \%$ & $6.0 \%$ & N/A & 5.0 & 14.0 \\
\hline Ngeow et al., 2010 $0^{[11]}$ & Singapore & 40 & Docetaxel alone & $36.7 \%$ & $0.0 \%$ & N/A & 4.5 & 12.8 \\
\hline Chua et al., 2000[38] & Hong Kong & 18 & Ifosfamide $+5 \mathrm{FU}+\mathrm{LV}$ (IFL) & $56.0 \%$ & $6.0 \%$ & 7.1 & 6.5 & N/A \\
\hline Altundag et al., 2004[39] & Turkey & 21 & Ifosphamide+Doxorubicin & $33.3 \%$ & $0.0 \%$ & N/A & 7.0 & N/A \\
\hline \multicolumn{9}{|l|}{ Targeted therapy } \\
\hline Elser et al., 2007[14] & Canada & 27 & Sorafenib alone & $40.7 \%$ & $0.0 \%$ & N/A & 1.8 & 4.2 \\
\hline Chan et al., 2005[8] & Hong Kong & 60 & Cetuximab+Carboplatin & $11.7 \%$ & $0.0 \%$ & N/A & 2.7 & 7.8 \\
\hline Chua et al., 2008 ${ }^{[40]}$ & Hong Kong & 19 & Gefitinib & $0.0 \%$ & $0.0 \%$ & N/A & 4.0 & 16.0 \\
\hline
\end{tabular}

Abbreviations: 5FU: 5-Flourouracil; LV: Leucovorin; N: Number of patient; ORR: Overall response rate; CR: Complete remission; mDR: Median duration of response; mTTP: Median time to p[rogression; mOS: Median overall survival; N/A: Not available from the original paper; UFUR: Tegafur-uracil; NPC: Nasopharyngeal carcinoma

for withdrawing from the study after one or two cycles of chemotherapy was patient's characteristics (3 poor compliances; 1 complete remission and request observation; $1 \mathrm{PR}$ but with prolonged thrombocytopenia as previously mentioned). None of them was classified to be related to the toxicity after confirmation.
Two $(9.1 \%)$ long-term survivors (more than 5 years) were documented in our study. The only common therapy after chemotherapy with the PULM regimen was radiotherapy, which was delivered for persistent localized metastatic lesions with curative intent. One of the patients had left femoral head single bone metastasis; however, the PULM regimen 
followed by local radiotherapy did not lead to regression in size of the lesion. Long-term survival was achieved after surgical removal. The other patient initially presented with early-stage NPC and achieved complete response after local radiotherapy. Single liver metastasis was found 2 years later. The PULM regimen resulted in a complete response; however, liver recurrence occurred 3 years after achievement of complete response. The patient underwent local radiotherapy plus systemic chemotherapy with gemcitabine, which resulted in complete response. In long-term survivors, effective local plus systemic treatment for patients with metastatic NPC has been discussed in the literature. ${ }^{[17,49]}$

There is one limitation in our study: The small number of patient. The main cause is slow recruitment. Decrease of failure events after primary chemo-radiotherapy in NPC patients and improvement of radiotherapy techniques (e.g., Intensity-modification radiotherapy, IMRT) in recent years are thought to be related. Another cause is that there was one concurrent competing trial (Gemcitabine plus cisplatin) during enrollment, which urged physicians to enroll patients into another trial. The initial design of this trial was alpha (0.05) with power of 0.90 when study sample reaches 42 (patients); however, the power decreases to 0.7638 by Fleming one stage procedure ${ }^{[26]}$ when the sample size was 22 (patients). Even though the power was not as significant as it was designed, the data still could provide evidence and help medical oncologists manage the efficacy, toxicities and limitations of PULM regimens in outpatient clinics.

In conclusion, the PULM regimen for patients with recurrent or metastatic NPC is an acceptable chemotherapy regimen in outpatient settings because of convenience and low toxic profile. We provide the results of this prospective phase II clinical trial for the evidence of PULM regimen, which demonstrated an overall response rate of $59.1 \%$ (including $13.6 \%$ complete remissions), a median duration of response of 15.9 months, progression-free of 10.0 months and overall survivals of 16.0 months.

\section{REFERENCES}

1. Lee AW, Foo W, Mang O, Sze WM, Chappell R, Lau WH, et al. Changing epidemiology of nasopharyngeal carcinoma in Hong Kong over a 20-year period (1980-99): An encouraging reduction in both incidence and mortality. Int J Cancer 2003;103:680-5.

2. Cancer Registry Annual Report in Taiwan Area, 2008, Bureau of Health Promotion, Taiwan Department of Health, R.O.C., 2010.

3. Au E, Ang PT. A phase II trial of 5-fluorouracil and cisplatinum in recurrent or metastatic nasopharyngeal carcinoma. Ann Oncol 1994;5:87-9.

4. Azli N, Fandi A, Bachouchi M, Rahal M, Lianes P, Wibault P, et al. Final report of a phase II study of chemotherapy with bleomycin, epirubicin, and cisplatin for locally advanced and metastatic/recurrent undifferentiated carcinoma of the nasopharyngeal type. Cancer J Sci Am 1995;1:222-9.
5. Foo KF, Tan EH, Leong SS, Wee JT, Tan T, Fong KW, et al. Gemcitabine in metastatic nasopharyngeal carcinoma of the undifferentiated type. Ann Oncol 2002;13:150-6.

6. Boussen H, Cvitkovic E, Wendling JL, Azli N, Bachouchi M, Mahjoubi R, et al. Chemotherapy of metastatic and/or recurrent undifferentiated nasopharyngeal carcinoma with cisplatin, bleomycin, and fluorouracil. J Clin Oncol 1991;9:1675-81.

7. Chi KH, Chan WK, Cooper DL, Yen SH, Lin CZ, Chen KY. A phase II study of outpatient chemotherapy with cisplatin, 5-fluorouracil, and leucovorin in nasopharyngeal carcinoma. Cancer 1994;73:247-52.

8. Chan AT, Hsu MM, Goh BC, Hui EP, Liu TW, Millward MJ, et al. Multicenter, phase II study of cetuximab in combination with carboplatin in patients with recurrent or metastatic nasopharyngeal carcinoma. J Clin Oncol 2005;23:3568-76.

9. Au E, Tan EH, Ang PT. Activity of paclitaxel by three-hour infusion in Asian patients with metastatic undifferentiated nasopharyngeal cancer. Ann Oncol 1998;9:327-9.

10. Ma BB, Tannock IF, Pond GR, Edmonds MR, Siu LL. Chemotherapy with gemcitabine-containing regimens for locally recurrent or metastatic nasopharyngeal carcinoma. Cancer 2002;95:2516-23.

11. Ngeow J, Lim WT, Leong SS, Ang MK, Toh CK, Gao F, et al. Docetaxel is effective in heavily pretreated patients with disseminated nasopharyngeal carcinoma. Ann Oncol 2011;22:718-22.

12. Dugan M, Choy D, Ngai A, Sham J, Choi P, Shiu W, et al. Multicenter phase II trial of mitoxantrone in patients with advanced nasopharyngeal carcinoma in Southeast Asia: An Asian-Oceanian Clinical Oncology Association Group study. J Clin Oncol 1993;11:70-6.

13. Zhang L, Zhang Y, Huang PY, Xu F, Peng PJ, Guan ZZ. Phase II clinical study of gemcitabine in the treatment of patients with advanced nasopharyngeal carcinoma after the failure of platinum-based chemotherapy. Cancer Chemother Pharmacol 2008;61:33-8.

14. Elser C, Siu LL, Winquist E, Agulnik M, Pond GR, Chin SF, et al. Phase II trial of sorafenib in patients with recurrent or metastatic squamous cell carcinoma of the head and neck or nasopharyngeal carcinoma. J Clin Oncol 2007;25:3766-73.

15. Taamma A, Fandi A, Azli N, Wibault P, Chouaki N, Hasbini A, et al. Phase II trial of chemotherapy with 5-fluorouracil, bleomycin, epirubicin, and cisplatin for patients with locally advanced, metastatic, or recurrent undifferentiated carcinoma of the nasopharyngeal type. Cancer 1999;86:1101-8.

16. Hong RL, Ting LL, Ko JY, Hsu MM, Sheen TS, Lou PJ, et al. Induction chemotherapy with mitomycin, epirubicin, cisplatin, fluorouracil, and leucovorin followed by radiotherapy in the treatment of locoregionally advanced nasopharyngeal carcinoma. J Clin Oncol 2001;19:4305-13.

17. Fandi A, Bachouchi M, Azli N, Taamma A, Boussen H, Wibault P, et al. Long-term disease-free survivors in metastatic undifferentiated carcinoma of nasopharyngeal type. J Clin Oncol 2000;18:1324-30.

18. Li CP, Chen JS, Chen LT, Yen CJ, Lee KD, Su WP, et al. A phase II study of weekly docetaxel and cisplatin plus oral tegafur/uracil and leucovorin as first-line chemotherapy in patients with locally advanced or metastatic gastric cancer. Br J Cancer 2010;103:1343-8.

19. Hortobagyi GN, Heim W, Hutchins L, Rivera E, Mason B, Booser DJ, et al. A phase 2 study of a fixed combination of uracil and ftorafur (UFT) and leucovorin given orally in a 3-times-daily regimen to treat patients with recurrent metastatic breast cancer. Cancer 2010;116:1440-5. 
20. Surmont V, Aerts JG, Pouw E, Tan KY, Vernhout R, Gras J, et al. Oral UFT, etoposide and leucovorin in recurrent non-small cell lung cancer: A non-randomized phase II study. Lung Cancer 2009;66:333-7.

21. Aykan NF, Idelevich E. The role of UFT in advanced gastric cancer. Ann Oncol 2008;19:1045-52.

22. Casado E, Pfeiffer P, Feliu J, Gonzalez-Baron M, Vestermark L, Jensen HA. UFT (tegafur-uracil) in rectal cancer. Ann Oncol 2008;19:1371-8.

23. Wang HM, Wang CH, Chang TC, Tsang NM, Liaw CT, Chen IH. Cisplatin, Tegafur plus Uracil (UFUR), and Leucovorin as a neoadjuvant chemotherapy for squamous cell carcinoma of the head and neck: A preliminary report of CGMH. Taipei, Taiwan: The $5^{\text {th }}$ International Conference of the Asian Clinical Oncology Society and the $6^{\text {th }}$ Taiwan Joint Cancer Conference, 2001.

24. Hong B, Lui VW, Hashiguchi M, Hui EP, Chan AT. Targeting tumor hypoxia in nasopharyngeal carcinoma. Head Neck 2013;35:133-45.

25. Chan Anthony TC, Teo Peter ML, Johnson PJ. Nasopharyngeal cancer. In: Bruce Brockstein and Bruce Brockstein. Head and Neck cancer, Ch. 11. $1^{\text {st }}$ ed. New York, Boston, Dordrecht, London, Moscow: Kluwer Academic Publishers; 2004. p. 284-5.

26. Fleming TR. One-sample multiple testing procedure for phase II clinical trials. Biometrics 1982;38:143-51.

27. Tannock IF, Osoba D, Stockler MR, Ernst DS, Neville AJ, Moore MJ, et al. Chemotherapy with mitoxantrone plus prednisone or prednisone alone for symptomatic hormone-resistant prostate cancer: A Canadian randomized trial with palliative end points. J Clin Oncol 1996;14:1756-64.

28. Ngan RK, Yiu HH, Lau WH, Yau S, Cheung FY, Chan TM, et al. Combination gemcitabine and cisplatin chemotherapy for metastatic or recurrent nasopharyngeal carcinoma: Report of a phase II study. Ann Oncol 2002;13:1252-8.

29. Chua DT, Sham JS, Au GK. A phase II study of docetaxel and cisplatin as first-line chemotherapy in patients with metastatic nasopharyngeal carcinoma. Oral Oncol 2005;41:589-95.

30. Yeo W, Leung TW, Leung SF, Teo PM, Chan AT, Lee WY, et al. Phase II study of the combination of carboplatin and 5-fluorouracil in metastatic nasopharyngeal carcinoma. Cancer Chemother Pharmacol 1996;38:466-70.

31. Yeo W, Leung TW, Chan AT, Chiu SK, Yu P, Mok TS, et al. A phase II study of combination paclitaxel and carboplatin in advanced nasopharyngeal carcinoma. Eur J Cancer 1998;34:2027-31.

32. Tan EH, Khoo KS, Wee J, Fong KW, Lee KS, Lee KM, et al. Phase II trial of a paclitaxel and carboplatin combination in Asian patients with metastatic nasopharyngeal carcinoma. Ann Oncol 1999;10:235-7.

33. Ciuleanu TE, Fountzilas G, Ciuleanu E, Plataniotis M, Todor N, Ghilezan N. Paclitaxel and carboplatin in relapsed or metastatic nasopharyngeal carcinoma: A multicenter phase II study. J BUON 2004;9:161-5.

34. Leong SS, Wee J, Rajan S, Toh CK, Lim WT, Hee SW, et al. Triplet combination of gemcitabine, paclitaxel, and carboplatin followed by maintenance 5-fluorouracil and folinic acid in patients with metastatic nasopharyngeal carcinoma. Cancer 2008;113:1332-7.

35. Wang CC, Chang JY, Liu TW, Lin CY, Yu YC, Hong RL. Phase II study of gemcitabine plus vinorelbine in the treatment of cisplatin-resistant nasopharyngeal carcinoma. Head Neck 2006;28:74-80.
36. Ma BB, Hui EP, Wong SC, Tung SY, Yuen KK, King A, et al. Multicenter phase II study of gemcitabine and oxaliplatin in advanced nasopharyngeal carcinoma--correlation with excision repair cross-complementing-1 polymorphisms. Ann Oncol 2009;20:1854-9.

37. Poon D, Chowbay B, Cheung YB, Leong SS, Tan EH. Phase II study of irinotecan (CPT-11) as salvage therapy for advanced nasopharyngeal carcinoma. Cancer 2005;103:576-81.

38. Chua DT, Kwong DL, Sham JS, Au GK, Choy D. A phase II study of ifosfamide, 5-fluorouracil and leucovorin in patients with recurrent nasopharyngeal carcinoma previously treated with platinum chemotherapy. Eur J Cancer 2000;36:736-41.

39. Altundag K, Aksoy S, Gullu I, Altundag O, Ozyar E, Yalcin S, et al. Salvage ifosfamide-doxorubicin chemotherapy in patients with recurrent nasopharyngeal carcinoma pretreated with Cisplatin-based chemotherapy. Med Oncol 2004;21:211-5.

40. Chua DT, Wei WI, Wong MP, Sham JS, Nicholls J, Au GK. Phase II study of gefitinib for the treatment of recurrent and metastatic nasopharyngeal carcinoma. Head Neck 2008;30:863-7.

41. Fandi A, Taamma A, Azli N, Bachouchi M, Yanes B, Armand JP, et al. Palliative treatment with low-dose continuous infusion 5-fluorouracil in recurrent and/or metastatic undifferentiated nasopharyngeal carcinoma type. Head Neck 1997;19:41-7.

42. Watanabe T, Sano M, Takashima S, Kitaya T, Tokuda Y, Yoshimoto M, et al. Oral uracil and tegafur compared with classic cyclophosphamide, methotrexate, fluorouracil as postoperative chemotherapy in patients with node-negative, high-risk breast cancer: National Surgical Adjuvant Study for Breast Cancer 01 Trial. J Clin Oncol 2009;27:1368-74.

43. Nakayama T, Noguchi S. Therapeutic usefulness of postoperative adjuvant chemotherapy with Tegafur-Uracil (UFT) in patients with breast cancer: Focus on the results of clinical studies in Japan. Oncologist 2010;15:26-36.

44. Chua D, Wei WI, Sham JS, Au GK. Capecitabine monotherapy for recurrent and metastatic nasopharyngeal cancer. Jpn J Clin Oncol 2008;38:244-9.

45. Li YH, Wang FH, Jiang WQ, Xiang XJ, Deng YM, Hu GQ, et al. Phase II study of capecitabine and cisplatin combination as first-line chemotherapy in Chinese patients with metastatic nasopharyngeal carcinoma. Cancer Chemother Pharmacol 2008;62:539-44.

46. Guigay J, Temam S, Bourhis J, Pignon JP, Armand JP. Nasopharyngeal carcinoma and therapeutic management: The place of chemotherapy. Ann Oncol 2006;17 Suppl 10:x304-7.

47. Ma BB, Chan AT. Recent perspectives in the role of chemotherapy in the management of advanced nasopharyngeal carcinoma. Cancer 2005;103:22-31.

48. Hasbini A, Mahjoubi R, Fandi A, Chouaki N, Taamma A, Lianes P, et al. Phase II trial combining mitomycin with 5-fluorouracil, epirubicin, and cisplatin in recurrent and metastatic undifferentiated carcinoma of nasopharyngeal type. Ann Oncol 1999;10:421-5.

49. Ma J, Wen ZS, Lin P, Wang X, Xie FY. The results and prognosis of different treatment modalities for solitary metastatic lung tumor from nasopharyngeal carcinoma: A retrospective study of 105 cases. Chin J Cancer 2010;29:787-95. 